\title{
Energy restriction as an antitumor target
}
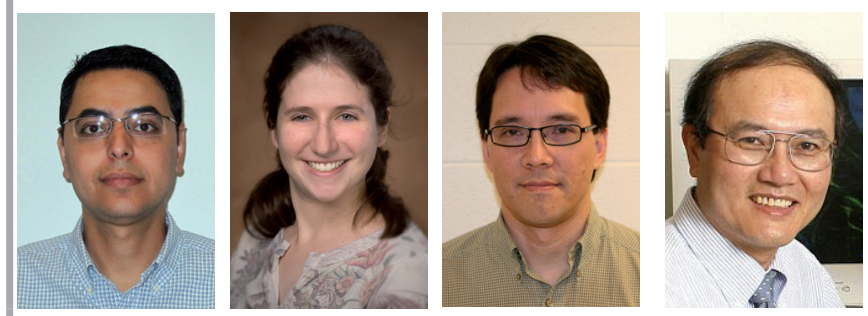

“...targeting tumor

metabolism has

emerged as a

promising therapeutic

... approach..."

Hany A Omar', Lisa Berman-Booty', Samuel K Kulp' \& Ching-Shih Chen ${ }^{\dagger 1}$

'Division of Medicinal Chemistry, College of Pharmacy \& Comprehensive Cancer Center, The Ohio State

University, Columbus, OH 43210, USA

tAuthor for correspondence: Tel.: +1 6746884008 - Fax: +1 6746888556 - chen.844@osu.edu

\section{Molecular basis for targeting tumor metabolism in cancer therapy}

The high need for energy in rapidly growing tumor cells links their survival to the robust availability of energy. In contrast to normal cells, transformed cells lack metabolic flexibility and switch to a glycolytic phenotype for energy production, an adaptive response to intermittent hypoxia that persists even in the presence of normal oxygen tension, the so-called Warburg effect [1-6]. This shift in energy production from oxidative phosphorylation to glycolysis is considered to be a fundamental property of cancer cells through the dysregulation of the pathways mediated by c-Myc and Akt [7-9]. This dependence on glycolysis for energy production has been linked to protection against the constitutive oxidative stress experienced by tumor cells [10-13]. The glycolytic switch can fuel the intracellular antioxidant machinery with reducing equivalents, such as NADPH, to raise tolerance to reactive oxygen species. In addition, the high rate of glycolytic flux associated with aerobic glycolysis enables tumor cells to divert intermediates of glycolysis into anabolic pathways for the synthesis of fatty acids, nucleotides and amino acids $[2,4,14]$. Recent evidence indicates that the glycolytic end product lactate serves as a biosynthetic intermediate for various cellular building blocks, thereby providing a growth advantage $[15,16]$.

\footnotetext{
"The emerging view of cancers as a metabolic disease reveals opportunities for the development of new therapeutic strategies."
}

The microenvironment-independent reliance on glycolysis and increased consumption of glucose render cancer cells more vulnerable to inhibition of glycolysis than normal cells, which can utilize diverse fuel sources for energy production.
Consequently, targeting tumor metabolism has emerged as a promising therapeutic or preventative approach, of which the proof of principle is evident from studies on the anticarcinogenic efficacy of chronic dietary energy restriction in various animal models [17-21]. Since chronic energy restriction proves to be difficult to implement as an antitumor strategy in humans, alternative approaches have been used to mimic the beneficial effects of energy restriction through interference with tumor metabolism. An early example of such an approach was nutritional metabolic therapy for brain cancer using the ketogenic diet, a high-fat, low-carbohydrate diet that shifts the prime substrate for energy metabolism from glucose to ketone bodies in order to disrupt tumor metabolism while maintaining the nutritional status of patients $[22,23]$.

\section{Tumor metabolism-targeted agents}

More recently, the development of small molecule agents that target various aspects of glucose metabolism has been the focus of many investigations, which are summarized in this article, according to cellular target.

\section{Glucose intake}

Glufosfamide, a covalent conjugate of glucose with an ifosfamide mustard, is preferentially taken up by cancer cells and then metabolized to release a cytotoxic compound, isophosphoramide. Glufosfamide has undergone clinical trials in solid tumors, alone or in combination with gemcitabine, with low-to-modest activities [24,25].

\section{Adenosine monophosphate-activated protein kinase}

Agents that activate adenosine monophosphateactivated protein kinase (AMPK) and, thus, induce cell cycle senescence include amino-imidazole-4-carboxamide ribonucleotide (AICAR)

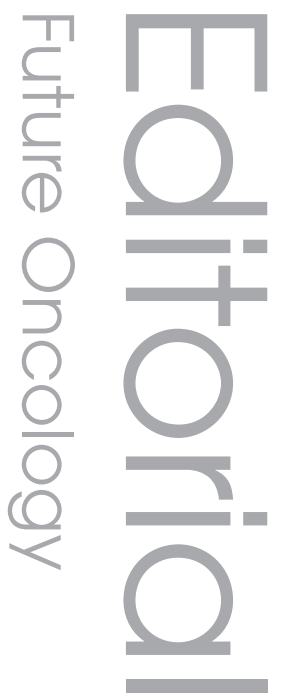

\footnotetext{
Keywords

- cancer therapeutic agent

- energy restriction = glycolysis

- tumor metabolism n Warburg

effect

future medicine $^{\text {pass }}$ fs
} 
and metformin and its analogs, phenformin and biguanide. AICAR is widely used experimentally to activate AMPK and inhibit the growth of tumor cells in vitro [26-28] and in vivo [26,27,29]. Epidemiologic data have suggested the chemopreventive potential of metformin in breast cancer $[30,31]$, which is supported by its efficacy in suppressing breast xenograft tumor growth in immunocompromised mice [32]. At the molecular level, metformin suppresses cancer cell growth by inhibiting mTOR-dependent translation initiation through AMPK activation [33,34] or by the phosphorylating and inactivating acetyl-CoA carboxylase, accompanied by the suppression of fatty acid synthase, which leads to growth inhibition by blocking lipogenesis [28,35].

\section{Glycolysis}

Inhibitors of glycolysis include resveratrol, a phytoalexin with widely reported anticancer activity, which is attributable, in part, to its energy restriction mimetic effects [36-40]. Resveratrol also activates AMPK [41,42]. While many studies have demonstrated resveratrol's anticancer activity in both in vitro and in vivo (largely in the context of chemoprevention) models [36,43], resveratrol shows no activity against the growth of existing tumors, except those of the skin and GI tract [44], which is, in part, caused by its poor systemic bioavailability [45-47]. OSU-CG12, a PPAR- $\gamma$ inactive ciglitazone derivative, is a new energy restriction mimetic agent with 20 - and 1000 -fold higher potency than resveratrol and 2-deoxyglucose (2-DG), respectively [39]. OSU-CG12 inhibits glucose metabolism through mechanisms at different molecular levels, including the cellular uptake of glucose, Akt signaling and the transcription of genes associated with glycolysis and energy metabolism. Investigation into additional mechanisms is ongoing.

\section{Hexokinase II}

Inhibition of the mitochondrial-bound hexokinase (HK) II by the indazole carboxylic acid lonidamine not only affects glucose metabolism, but also sensitizes cancer cells to apoptosis by facilitating the docking of Bax on the voltage-dependent anion channel, a HK II binding partner [48]. In Phase II trials, the combination of lonidamine and cytotoxic chemotherapy was active against advanced non-small-cell lung cancer and ovarian cancer [49,50]. However, as a single agent, lonidamine showed little activity against non-small-cell lung cancer or glioblastoma multiforme [51,52]. 3-bromopyruvate, another HK II inhibitor, is an alkylating agent with structural similarity to lactate, which may enter cancer cells on the same transporter that exports lactate, and then induce ATP depletion [53]. 3-bromopyruvate has demonstrated suppressive activities against hepatocellular carcinoma [54] and breast cancer [55], both in vitro and in vivo. 2-DG also inhibits HK II activity [56].

\section{Phosphohexose isomerase}

2-deoxyglucose blocks glycolysis through the inhibition of phosphohexose isomerase [57,58], leading to a depletion of ATP and glucose derivatives required for protein glycosylation. 2-DG also induces the unfolded protein response, as does low glucose stress [59,60], and inhibits the growth of rat fibrosarcoma [61], hepatocellular carcinoma $[62,63]$ and other tumors, as a single agent or in combination with chemotherapeutics $[64,65]$.

\footnotetext{
"Further understanding of the signaling

mechanisms underlying the antitumor

effects of these tumor metabolism-

targeted agents will help foster novel strategies for cancer therapy."
}

\section{Pyruvate kinase}

TLN-232 (or CAP-232) is a cyclic heptapeptide that targets the M2 splice isoform of pyruvate kinase, which has been reported to play an important role in cancer metabolism and tumor growth [66]. TLN-232 is currently undergoing a small Phase II study in metastatic melanoma [67].

\section{Lactate dehydrogenase $\mathrm{A}$}

The treatment of P493 human lymphoma B cells with FX11, an inhibitor of lactate dehydrogenase A, reduces ATP levels and causes oxidative stress-induced cell death, and, thus, inhibits the progression of human lymphoma and pancreatic xenograft tumor growth [68].

\section{Pyruvate dehydrogenase kinase}

Dichloroacetate has been proposed as a novel and relatively nontoxic anticancer agent that can reverse the glycolytic phenotype in cancer cells through the inhibition of pyruvate dehydrogenase kinase [69]. Dichloroacetate has been used clinically for the treatment of lactic acidosis since 1969, and is currently undergoing clinical trials to evaluate its toxicity in cancer patients.

\section{Monocarboxylate transporter 1}

Inhibition of monocarboxylate transporter 1 by $\alpha$-cyano-4-hydroxy-cinnamate induces a switch from lactate-fueled respiration to 
glycolysis in oxygenated tumor cells, and, thus, suppresses lung and colon xenograft tumor growth [70].

\section{ATP citrate lyase}

The inhibition of ATP citrate lyase by SB2499 blocks cytosolic acetyl-CoA production and lipid synthesis, thereby inhibiting proliferation and survival of tumor cells displaying aerobic glycolysis in vitro and in vivo [71].

\section{Opportunities \& challenges}

The emerging view of cancers as a metabolic disease reveals opportunities for the development of new therapeutic strategies. Many of the tumor metabolism-targeted agents described in this article exhibit in vivo efficacy alone or in combination with chemotherapeutic drugs in advanced cancers.

It is generally believed that interfering with energy metabolism causes ATP depletion and metabolic stress, leading to cell death. However, data from ours and other laboratories indicate that reducing glycolytic rate by energy restriction activates multiple signaling pathways, including those mediated by the $\mathrm{NAD}^{+}$-dependent histone deacetylase sirtuin 1, AMPK and endoplasmic reticulum stress [39]. This complicated signaling network affects many aspects of cellular functions relevant to cell cycle regulation, survival and aggressive phenotype, culminating in cancer cell death through autophagy and apoptosis. Therefore, it is plausible to achieve synergy with other molecularly targeted agents, such as kinase inhibitors or histone deacetylase inhibitors, to kill cancer cells. Further understanding of the signaling mechanisms underlying the antitumor effects of these tumor metabolismtargeted agents will help foster novel strategies for cancer therapy.

Despite substantial advances in the preclinical development of these tumor metabolism-targeted agents, a number of issues warrant further investigation. Toxicity is common among many drugs associated with high doses required to attain in vivo efficacy and/or off-target pharmacological activities. For example, the US FDA has suspended the clinical trial of 2-DG for advanced prostate cancer (NCT00633087), and that of lonidamine for benign prostate hypertrophy owing to hepatic side effects [101]. In addition, not all cancer cells are susceptible to interference with energy metabolism. AMPK activators, such as AICAR and metformin, were reported to selectively inhibit p53-deficient tumor cell growth, suggesting that AMPK activation forces a metabolic conversion that $\mathrm{p} 53^{-/-}$ cells are unable to execute [29]. Therefore, it is important to define the determining factor for the resistant phenotype for each type of tumor metabolism-targeted agent.

Financial \& competing interests disclosure
This work is supported by Public Health Service grant
CA112250 from the National Cancer Institute,
Department of Defense Prostate Cancer Research
Program grant W81XWH-09-0198 and T-32
Institutional National Research Service Award in
Mouse Models of Human Disease (RL Kirschstein). The
authors have no other relevant affiliations or financial
involvement with any organization or entity with a
financial interest in or financial conflict with the sub-
ject matter or materials discussed in the manuscript
apart from those disclosed. No writing assistance was
utilized in the production of this manuscript.

This work is supported by Public Health Service grant CA112250 from the National Cancer Institute, Department of Defense Prostate Cancer Research Program grant W81XWH-09-0198 and T-32 Institutional National Research Service Award in Mouse Models of Human Disease (RL Kirschstein). The authors have no other relevant affiliations or financial involvement with any organization or entity with a financial interest in or financial conflict with the subject matter or materials discussed in the manuscript utilized in the production of this manuscript.

\section{Bibliography}

1. Hsu PP, Sabatini DM: Cancer cell metabolism: Warburg and beyond. Cell 134(5), 703-707 (2008).

2. Kaelin WG Jr, Thompson CB: Q\&A: cancer: clues from cell metabolism. Nature 465(7298), 562-564 (2010).

3. Kim JW, Dang CV: Cancer's molecular sweet tooth and the Warburg effect. Cancer Res. 66(18), 8927-8930 (2006).

4. Kroemer G, Pouyssegur J: Tumor cell metabolism: cancer's Achilles' heel. Cancer Cell 13(6), 472-482 (2008).

5. Seyfried TN, Shelton LM: Cancer as a metabolic disease. Nutr. Metab. (Lond.) 7, 7 (2010).
6. Zhivotovsky B, Orrenius S: The Warburg effect returns to the cancer stage. Semin. Cancer Biol. 19(1), 1-3 (2009).

7. Dang CV: c-Myc target genes involved in cell growth, apoptosis, and metabolism. Mol. Cell. Biol. 19(1), 1-11 (1999).

8. Elstrom RL, Bauer DE, Buzzai M et al.: Akt stimulates aerobic glycolysis in cancer cells. Cancer Res. 64(11), 3892-3899 (2004).

9. Garber K: Energy boost: the Warburg effect returns in a new theory of cancer. J. Natl Cancer Inst. 96(24), 1805-1806 (2004).

10. Soucek T, Cumming R, Dargusch R, Maher P, Schubert D: The regulation of glucose metabolism by HIF-1 mediates a neuroprotective response to amyloid- $\beta$ peptide. Neuron 39(1), 43-56 (2003).
11. Guo S, Bragina O, Xu Y et al.: Glucose up-regulates HIF-1 $\alpha$ expression in primary cortical neurons in response to hypoxia through maintaining cellular redox status. J. Neurochem. 105(5), 1849-1860 (2008).

12. Pani G, Colavitti R, Bedogni B et al.: Mitochondrial superoxide dismutase: a promising target for new anticancer therapies. Curr. Med. Chem. 11(10), 1299-1308 (2004).

13. Hempel N, Ye H, Abessi B, Mian B, Melendez JA: Altered redox status accompanies progression to metastatic human bladder cancer. Free Radic. Biol. Med. 46(1), 42-50 (2009).

14. Vander Heiden MG, Cantley LC, Thompson CB: Understanding the Warburg effect: the metabolic requirements of cell proliferation. Science 324, 1029-1033 (2009). 
15. Bui T, Thompson CB: Cancer's sweet tooth. Cancer Cell 9 (6), 419-420 (2006).

16. Fantin VR, St-Pierre J, Leder P: Attenuation of LDH-A expression uncovers a link between glycolysis, mitochondrial physiology, and tumor maintenance. Cancer Cell 9(6), 425-434 (2006).

17. Jiang W, Zhu Z, Thompson HJ: Dietary energy restriction modulates the activity of AMP-activated protein kinase, Akt, and mammalian target of rapamycin in mammary carcinomas, mammary gland, and liver. Cancer Res. 68(13), 5492-5499 (2008).

18. Hursting SD, Lavigne JA, Berrigan D, Perkins SN, Barrett JC: Calorie restriction, aging, and cancer prevention: mechanisms of action and applicability to humans. Annu. Rev. Med. 54, 131-152 (2003).

19. Berrigan D, Perkins SN, Haines DC, Hursting SD: Adult-onset calorie restriction and fasting delay spontaneous tumorigenesis in p53-deficient mice. Carcinogenesis 23(5), 817-822 (2002).

20. Mukherjee P, Sotnikov AV, Mangian HJ et al.: Energy intake and prostate tumor growth, angiogenesis, and vascular endothelial growth factor expression. J. Natl Cancer Inst. 91(6), 512-523 (1999).

21. Kritchevsky D: Caloric restriction and experimental carcinogenesis. Toxicol. Sci. 52(Suppl. 2), 13-16 (1999).

22. Hartman AL, Vining EP: Clinical aspects of the ketogenic diet. Epilepsia 48(1), 31-42 (2007).

23. Nebeling LC, Miraldi F, Shurin SB, Lerner E: Effects of a ketogenic diet on tumor metabolism and nutritional status in pediatric oncology patients: two case reports. J. Am. Coll. Nutr. 14(2), 202-208 (1995).

24. Chiorean EG, Dragovich T, Hamm J et al.: A Phase 1 dose-escalation trial of glufosfamide in combination with gemcitabine in solid tumors including pancreatic adenocarcinoma. Cancer Chemother. Pharmacol. 61(6), 1019-1026 (2008).

25. Ciuleanu TE, Pavlovsky AV, Bodoky G et al: A randomised Phase III trial of glufosfamide compared with best supportive care in metastatic pancreatic adenocarcinoma previously treated with gemcitabine. Eur. J. Cancer 45(9), 1589-1596 (2009).

26. Swinnen JV, Beckers A, Brusselmans K et al.: Mimicry of a cellular low energy status blocks tumor cell anabolism and suppresses the malignant phenotype. Cancer Res. 65(6), 2441-2448 (2005).

27. Rattan R, Giri S, Singh AK, Singh I: 5-aminoimidazole-4-carboxamide-1- $\beta$-Dribofuranoside inhibits cancer cell proliferation in vitro and in vivo via AMP-activated protein kinase. J. Biol. Chem. 280 (47), 39582-39593 (2005).

28. Xiang X, Saha AK, Wen R, Ruderman NB, Luo Z: AMP-activated protein kinase activators can inhibit the growth of prostate cancer cells by multiple mechanisms. Biochem. Biophys. Res. Commun. 321(1), 161-167 (2004).

29. Buzzai M, Jones RG, Amaravadi RK et al.: Systemic treatment with the antidiabetic drug metformin selectively impairs $p 53$-deficient tumor cell growth. Cancer Res. 67(14), 6745-6752 (2007).

30. Evans JM, Donnelly LA, Emslie-Smith AM, Alessi DR, Morris AD: Metformin and reduced risk of cancer in diabetic patients. BMJ 330 (7503), 1304-1305 (2005).

31. Bodmer M, Meier C, Krahenbuhl S, Jick SS, Meier CR: Long-term metformin use is associated with decreased risk of breast cancer. Diabetes Care 33(6), 1304-1308 (2010).

32. Liu B, Fan Z, Edgerton SM et al.: Metformin induces unique biological and molecular responses in triple negative breast cancer cells. Cell Cycle 8(13), 2031-2040 (2009).

33. Dowling RJ, Zakikhani M, Fantus IG, Pollak M, Sonenberg N: Metformin inhibits mammalian target of rapamycin-dependent translation initiation in breast cancer cells. Cancer Res. 67(22), 10804-10812 (2007).

34. Zakikhani M, Dowling R, Fantus IG, Sonenberg N, Pollak M: Metformin is an AMP kinase-dependent growth inhibitor for breast cancer cells. Cancer Res. 66(21), 10269-10273 (2006).

35. Guo D, Hildebrandt IJ, Prins RM et al.: The AMPK agonist AICAR inhibits the growth of EGFRvIII-expressing glioblastomas by inhibiting lipogenesis. Proc. Natl Acad. Sci. USA 106(31), 12932-12937 (2009).

36. Baur JA, Sinclair DA: Therapeutic potential of resveratrol: the in vivo evidence. Nat. Rev. Drug Discov. 5(6), 493-506 (2006).

37. Cucciolla V, Borriello A, Oliva A et al:: Resveratrol: from basic science to the clinic. Cell Cycle 6(20), 2495-2510 (2007).

38. Bishayee A: Cancer prevention and treatment with resveratrol: from rodent studies to clinical trials. Cancer Prev. Res. (Phila.) 2(5), 409-418 (2009).

39. Wei S, Kulp SK, Chen CS: Energy restriction as an antitumor target of thiazolidinediones. J. Biol. Chem. 285(13), 9780-9791 (2010).

40. Lin JN, Lin VC, Rau KM et al:: Resveratrol modulates tumor cell proliferation and protein translation via SIRT1-dependent AMPK activation. J. Agric. Food Chem. 58(3), 1584-1592 (2010).
41. Athar M BJ, Kopelovich L, Bickers DR, Kim AL: Multiple molecular targets of resveratrol: anti-carcinogenic mechanisms. Arch. Biochem. Biophys. 486, 95-102 (2009).

42. Hwang JT, Kwak DW, Lin SK, Kim HM, Kim YM, Park OJ: Resveratrol induces apoptosis in chemoresistant cancer cells via modulation of AMPK signaling pathway. Ann. NY Acad. Sci.1095, 441-448 (2007).

43. Jang M, Cai L, Udeani GO et al.: Cancer chemopreventive activity of resveratrol, a natural product derived from grapes. Science 275(5297), 218-220 (1997).

44. Athar M, Back JH, Tang X et al.: Resveratrol: a review of preclinical studies for human cancer prevention. Toxicol. Appl. Pharmacol. 224(3), 274-283 (2007).

45. Boocock DJ, Faust GE, Patel KR et al.: Phase I dose escalation pharmacokinetic study in healthy volunteers of resveratrol, a potential cancer chemopreventive agent. Cancer Epidemiol. Biomarkers Prev. 16(6), 1246-1252 (2007).

46. Wenzel E, Soldo T, Erbersdobler H, Somoza V: Bioactivity and metabolism of trans-resveratrol orally administered to Wistar rats. Mol. Nutr. Food Res. 49(5), 482-494 (2005).

47. Niles RM, Cook CP, Meadows GG et al.: Resveratrol is rapidly metabolized in athymic (nu/nu) mice and does not inhibit human melanoma xenograft tumor growth. J. Nutr. 136(10), 2542-2546 (2006).

48. Mathupala SP, Ko YH, Pedersen PL: Hexokinase II: cancer's double-edged sword acting as both facilitator and gatekeeper of malignancy when bound to mitochondria. Oncogene 25(34), 4777-4786 (2006).

49. De Lena M, Lorusso V, Latorre A et al.: Paclitaxel, cisplatin and lonidamine in advanced ovarian cancer. A Phase II study. Eur. J. Cancer 37(3), 364-368 (2001).

50. Portalone L, Lombardi A, Antilli A et al:: Treatment of inoperable non-small cell lung carcinoma stage IIIb and IV with cisplatin, epidoxorubicin, vindesine and lonidamine: a Phase II study. Tumori 85(4), 239-242 (1999).

51. De Marinis F, Rinaldi M, Ardizzoni A et al.: The role of vindesine and lonidamine in the treatment of elderly patients with advanced non-small cell lung cancer: a Phase III randomized FONICAP trial. Italian Lung Cancer Task Force. Tumori 85(3), 177-182 (1999).

52. Oudard S, Carpentier A, Banu E et al.: Phase II study of lonidamine and diazepam in the treatment of recurrent glioblastoma multiforme. J. Neurooncol. 63(1), 81-86 (2003). 
53. Ko YH, Smith BL, Wang Y et al.: Advanced cancers: eradication in all cases using 3-bromopyruvate therapy to deplete ATP. Biochem. Biophys. Res. Commun. 324(1), 269-275 (2004).

54. Kim W, Yoon JH, Jeong JM et al.: Apoptosis-inducing antitumor efficacy of hexokinase II inhibitor in hepatocellular carcinoma. Mol. Cancer Ther. 6(9), 2554-2562 (2007).

55. Buijs M, Vossen JA, Geschwind JF et al.: Specificity of the anti-glycolytic activity of 3-bromopyruvate confirmed by FDG uptake in a rat model of breast cancer. Invest. New Drugs 27(2), 120-123 (2009).

56. Chen Z, Lu W, Garcia-Prieto C, Huang P: The Warburg effect and its cancer therapeutic implications. J. Bioenerg. Biomembr. 39, 267-274 (2007).

57. Sols A, Crane RK: Substrate specificity of brain hexokinase. J. Biol. Chem. 210(2), 581-595 (1954).

58. Tower DB: The effects of 2-deoxy-D-glucose on metabolism of slices of cerebral cortex incubated in vitro. J. Neurochem. 3(2), 185-205 (1958).

59. Hightower LE: Stress proteins in biology and medicine. In: Cold Spring Harbor Monograph Series 19. Morimoto RI, Tissieres A, Georgopoulos C (Eds). Cold Spring Harbor Laboratory, NY, USA, 572-573 (1990).
60. Little E, Ramakrishnan M, Roy B, Gazit G, Lee AS: The glucose-regulated proteins (GRP78 and GRP94): functions, gene regulation, and applications. Crit. Rev. Eukaryot. Gene Expr. 4(1), 1-18 (1994).

61. Kern KA, Norton JA: Inhibition of established rat fibrosarcoma growth by the glucose antagonist 2-deoxy-D-glucose. Surgery 102(2), 380-385 (1987).

62. Cay O, Radnell M, Jeppsson B, Ahren B, Bengmark S: Inhibitory effect of 2-deoxy-Dglucose on liver tumor growth in rats. Cancer Res. 52(20), 5794-5796 (1992).

63. Geschwind JF, Georgiades CS, Ko YH, Pedersen PL: Recently elucidated energy catabolism pathways provide opportunities for novel treatments in hepatocellular carcinoma. Expert Rev. Anticancer. Ther. 4(3), 449-457 (2004).

64. Maher JC, Krishan A, Lampidis TJ: Greater cell cycle inhibition and cytotoxicity induced by 2-deoxy-D-glucose in tumor cells treated under hypoxic vs aerobic conditions. Cancer Chemother. Pharmacol. 53(2), 116-122 (2004).

65. Maschek G, Savaraj N, Priebe W et al.: 2-deoxy-D-glucose increases the efficacy of adriamycin and paclitaxel in human osteosarcoma and non-small cell lung cancers in vivo. Cancer Res. 64(1), 31-34 (2004).
66. Christofk HR, Vander Heiden MG, Harris MH et al:: The M2 splice isoform of pyruvate kinase is important for cancer metabolism and tumour growth. Nature 452(7184), 230-233 (2008).

67. Hersey P, Watts RN, Zhang XD, Hackett J: Metabolic approaches to treatment of melanoma. Clin. Cancer Res. 15(21), 6490-6494 (2009).

68. Le A, Cooper CR, Gouw AM et al.: Inhibition of lactate dehydrogenase A induces oxidative stress and inhibits tumor progression. Proc. Natl Acad. Sci. USA 107(5), 2037-2042 (2010).

69. Michelakis ED, Webster L, Mackey JR: Dichloroacetate (DCA) as a potential metabolic-targeting therapy for cancer. Br. J. Cancer 99(7), 989-994 (2008).

70. Sonveaux P, Vegran F, Schroeder T et al: : Targeting lactate-fueled respiration selectively kills hypoxic tumor cells in mice. J. Clin. Invest. 118(12), 3930-3942 (2008).

71. Hatzivassiliou G, Zhao F, Bauer DE et al:: ATP citrate lyase inhibition can suppress tumor cell growth. Cancer Cell 8(4), 311-321 (2005).

\section{Website}

101. Suspension of lonidamine clinical trial www.nelm.nhs.uk/en/NeLM-Area/ News/487372/487504/487505 